\title{
Research investigating antioxidation of astaxanthin extracted from Haematoccus pluvialis in mice
}

\author{
Shaolei Yu MSc¹, Weichun Du BD¹, Qianwen Li MSc²
}

S Yu, W Du, Q Li. Research investigating antioxidation of astaxanthin extracted from Haematoccus pluvialis in mice. Curr Res Integr Med 2015;1(3):37-38.

OBJECTIVE: To evaluate the antioxidative effect of astaxanthin extracted from Haematoccus pluvialis in mice.

METHODS: Fifty mice were randomly divided into five groups (high-dose [astaxanthin $4.0 \mathrm{mg} / \mathrm{kg}$; medium dose [astaxanthin $2.0 \mathrm{mg} / \mathrm{kg}$ ]; low dose [astaxanthin $1.0 \mathrm{mg} / \mathrm{kg}$ ]; model control; and blank control). The experimental substance was administered every day. Mice in the model group and the control group received an equal volume of vegetable oil for 30 days. After 30 days, blood antioxidant enzyme activity was measured in tail blood. Except for the blank control group, the other groups were exposed to $8 \mathrm{~Gy}{ }^{60} \mathrm{Co}$ irradiation. On the fourth day after irradiation, all animals were euthanized and liver tissue was tested for malondialdehyde, superoxide dismutase and glutathione peroxidase activity levels.

RESULTS: Superoxide dismutase levels in all three groups that received astaxanthin were significantly higher than in the model control group, and malondialdehyde levels in all three groups that received astaxanthin were significantly lower than in the control group. Glutathione peroxidase enzyme activity in the high-dose group was significantly higher in the model control group.

CONCLUSION: According to the evaluation standards in Inspection and Assessment Standard for Health Food, 2003 edition, the present study demonstrated that astaxanthin extracted from Haematoccus pluvialis has antioxidative effects in a mouse model.

Key Words: Antioxidation; Astaxanthin; Haematococcus pluvialis; Mice

\section{Dose group}

Fifty mice were randomly divided into five groups (high-dose [astaxanthin $4.0 \mathrm{mg} / \mathrm{kg}$ ], medium dose [astaxanthin $2.0 \mathrm{mg} / \mathrm{kg}$ ], low-dose [astaxanthin $1.0 \mathrm{mg} / \mathrm{kg}$, model control and blank control). The amount of astaxanthin administered to the groups receiving astaxanthin, repectively, were equivalent to $20 \times, 10 \times$ and $5 \times$ the human recommended amount.

\section{Experimental methods}

The three groups receiving aztaxanthin were administered treatment according to the corresponding dose for 30 days. The model control group and blank control group were given equal volumes of vegetable oil for 30 days. After 30 days, blood samples taken from the tail were tested for antioxidant enzyme activity. In addition to the blank control group, each group was exposed to $8 \mathrm{~Gy}{ }^{60} \mathrm{Co} \gamma$ ray exposure once. Four days after irradiation, all animals were euthanized to test for MDA content, SOD activity and GSH-Px in liver tissue according to the corresponding kit instructions.

\section{Statistical analysis}

ANOVA was performed using PEMS 3.0 (Chinese Medical Encyclopedia Medical Statistics, China). University (China). The mice were quarantined for one week before the experiment. The mice were fed in a barrier level animal room, which was mainted at a temperature between $20^{\circ} \mathrm{C}$ and $23^{\circ} \mathrm{C}$, and a relative humidity between $55 \%$ and $65 \%$ for the duration of the experiment. Mice were provided with food and water ad libitum.

\section{Equiptment and reagents}

For the present study, the following equiptment was used: 722 spectrophotometer; water bath maintained at a constant temperature; microadjustable pipette; high- and low-speed centrifuges; vortex mixers; a DY89-1 electric glass homogenizer; and malondialdehyde (MDA), superoxide dismutase (SOD) and glutathione peroxidase (GSH-Px) assay kits (Nanjing Jiancheng Bioengineering Institute, China). Chloroform, ethanol, acetic acid and other chemical reagents were analytical grade.

\section{RESULTS}

Effect of astaxanthin on body weight of mice (Table 1).

SOD activity in mice before irradiation

There was no significant difference among any of the groups in SOD activity before irradiation $(\mathrm{P}>0.05)$ (Table 2$)$.

Effect of astaxanthin MDA, SOD and GSH-Px activity in liver tissue The activity of MDA in the model group was significantly higher than that of the control group $(\mathrm{P}<0.05)$, and MDA content of the three
There were no adverse effects on body weight of the mice $(\mathrm{P}>0.05)$

\footnotetext{
${ }^{1}$ Yunnan Green A Biological Project Co., LTD, Kunming 650106; 2 College of Pharmacy, JiNan University, Guangzhou 510632, China Correspondence: Shaolei Yu, Yunnan Green A Biological Project Co., LTD, 1088 Haiyuan Zhong Road Kunming High-Develop Zone, Yunnan, Kunming 650106, China. Telephone 13529390463, e-mail 15980819@163.com
} 
TABLE 1

Effect of astaxanthin on the body weight of mice

\begin{tabular}{lcccc}
\hline & & \multicolumn{3}{c}{ Weight, $\mathbf{~}$} \\
\cline { 3 - 5 } Group & $\mathbf{n}$ & Starting & $\begin{array}{c}\text { Mid- } \\
\text { experiment }\end{array}$ & Final \\
\hline Blank control & 10 & $20.5 \pm 2.0$ & $33.7 \pm 3.1$ & $42.3 \pm 3.9$ \\
Model & 10 & $20.2 \pm 2.3$ & $33.2 \pm 3.2$ & $43.1 \pm 4.0$ \\
Low dose & 10 & $20.3 \pm 2.3$ & $33.5 \pm 3.8$ & $44.1 \pm 3.3$ \\
Medium dose & 10 & $20.3 \pm 1.7$ & $34.1 \pm 4.4$ & $44.9 \pm 3.7$ \\
High dose & 10 & $20.6 \pm 1.9$ & $33.8 \pm 2.9$ & $43.5 \pm 3.1$ \\
\hline
\end{tabular}

Data presented as mean $\pm S D$ unless otherwise indicated

\section{TABLE 2}

Superoxide dismutase (SOD) activity in mice before irradiation

\begin{tabular}{lcc}
\hline Group & $\mathbf{n}$ & SOD activity, NU/mL \\
\hline Blank control & 10 & $3143.6 \pm 198.6$ \\
Model & 10 & $3054.2 \pm 267.6$ \\
Low-dose & 10 & $3038.6 \pm 242.0$ \\
Medium dose & 10 & $3198.9 \pm 250.5$ \\
High-dose & 10 & $3228.7 \pm 232.5$ \\
\hline
\end{tabular}

Data presented as mean $\pm S D$ unless otherwise indicated

TABLE 3

Effect of astaxanthin on malondialdehyde (MDA), superoxide dismutase (SOD), glutathione peroxidase (GSH-Px) activities in liver tissue

\begin{tabular}{lcccl}
\hline Group & $\mathbf{n}$ & $\begin{array}{c}\text { MDA activity, } \\
\text { nmol/g liver wet } \\
\text { weight }\end{array}$ & $\begin{array}{c}\text { SOD activity, } \\
\text { nmol/g liver wet } \\
\text { weight }\end{array}$ & $\begin{array}{c}\text { GSH-Px, } \\
\text { activity units/g } \\
\text { liver wet weight }\end{array}$ \\
\hline Blank control & 10 & $110.2 \pm 46.4$ & $33579.5 \pm 885.8$ & $4088.0 \pm 703.2$ \\
Model & 10 & $190.6 \pm 73.0^{\star}$ & $27853.8 \pm 1566.4^{\star}$ & $1950.0 \pm 1028.5^{\star}$ \\
Low dose & 10 & $130.6 \pm 40.0^{\dagger}$ & $31999.0 \pm 969.8^{\dagger}$ & $2048.0 \pm 730.4$ \\
Medium dose & 10 & $123.3 \pm 38.1^{\dagger}$ & $31983.5 \pm 921.7^{\dagger}$ & $2708.0 \pm 1461.7$ \\
High dose & 10 & $104.4 \pm 42.3^{\dagger}$ & $32595.6 \pm 984.2^{\dagger}$ & $3524.0 \pm 810.9^{\dagger}$ \\
\hline
\end{tabular}

Data presented as mean $\pm S D$ unless otherwise indicated. *Significant difference compared with the control group $(P<0.01)$; ${ }^{\dagger}$ Significant difference compared with the control group $(P<0.05)$

groups that received antaxanthin were significantly lower than in the control group $(\mathrm{P}<0.05)$. SOD activity in the model group was significantly lower than in the control group $(\mathrm{P}<0.05)$, and SOD activity of the three groups that received astaxanthin were significantly higher than in the control group $(\mathrm{P}<0.05)$. The GSH-Px activity in the model group was significantly lower than in the control group $(\mathrm{P}<0.05)$, and the GSH-Px activity in the high-dose group was significantly higher than in the model control group $(\mathrm{P}<0.05)$ (Table 3$)$.

\section{DISCUSSION}

Astaxanthin (3,3'-hydroxy- $\beta$, $\beta^{\prime}$-carotene-4, $4^{\prime}$-dione $)$ is a type of shortchain antioxidant that has strong antioxidant properties. It can provide an electron or a radical to react with another radical, which can then absorb excess energy, making the radical transform to a nonactive or more stable compound, thereby interrupting the radical chain reaction process. The main activity of its antioxidant mechanism has several aspects (4-6):

1. Quenching singlet oxygen and scavenging oxygen-free radicals: astaxanthin has a strong ability to quench singlet oxygen capacity. Its molecular structure, which contains both hydroxyl and keto groups, may also promote keto-hydroxyl hydrogen transfer to peroxide radicals;

2. Stabilizing membrane structure and reducing membrane fluidity: astaxanthin contains polar ends, which act as bridge-like molecules across the cell membrane, which can increase its stability and mechanical strength, and can reduce membrane permeability, limiting penetration of intracellular oxidants and protecting cells from oxidative damage;

3. Increasing antioxidant enzyme activity: astaxanthin can act as an antioxidation system by activating the cells to decrease MDA production, reducing its concentration in the body, and increase $\mathrm{SOD}$ and GSH-Px activity, protecting cells from free radicalinduced oxidative damage;

4. Reducing the oxidative damage to mitochondria: after hydrogen peroxide damage, the contents of MDA and NO increased, while the activities of GSH, SOD, ATP enzyme decreased in mitochondria. Astaxanthin can reverse these changes.

5. Inhibition of lipid peroxidation: astaxanthin inhibits unsaturated fatty-acid methyl ester peroxide, phosphatidylcholine protection from oxidation, delay time phosphatidylcholine single large bubble (liposome) peroxidation.

Astaxanthin extracted from Haematoccus pluvialis using supercritical $\mathrm{CO}_{2}$ extraction technology, compared with synthetic astaxanthin, has more stable and secure advantages. The results of the present study demonstrate that SOD activity in the three groups that received astaxanthin were significantly higher than in the model control group. MDA activity in the three groups that received astaxanthin was significantly lower than in the model group. Finally, GSH-Px activity in the high-dose group was significantly higher than the model control group. According to the Inspection and Assessment Standard for Health Food (2003 edition), the results produced by astaxanthin extracted from Haematoccus pluvialis suggest that it produced antioxidant effects.

\section{REFERENCES}

1. Huang W, Hong B, Yi R. Astaxanthin production methods and biological activity research progress. Chinese Food Additives 2012;6:214-8.

2. Wang C, Han S, Chen Z. Haematococcus pluvialis antioxidant system of active oxygen scavenge mechanism. Aquatic Biology 2012:36:804-8

3. $\mathrm{Xu} \mathrm{H}, \mathrm{Cao} \mathrm{B}$, Chen J. Biological function of astaxanthin and its application. Feed Wide-Angle 2012;11:23-5.

4. Leite MF, Massuyama MM, Otton R. Astaxanthin restores the enzymatic antioxidant profile in salivary gland of alloxan-induced diabetic rats. Archives of Oral Biology 2010;55:479-85.

5. Lee DH, Kim CS, Lee YJ. Astaxanthin protects against MPTP/ $\mathrm{MPP}+$-induced mitochondrial dysfunction and ROS production in vivo and in vitro. Food Chem Toxicol 2011;49:271-80.

6. Yang Y, Zhou Y, Xu H. Animal studies astaxanthin antioxidant. Modern Preventive Medicine 2009;36:2432-3. 